\title{
A Zipper-Like On/Off-Switchable Molecularly Imprinted Polymer
}

\author{
Songjun Li, Yi Ge, Sergey A Piletsky and Anthony Turner
}

\section{Linköping University Post Print}

N.B.: When citing this work, cite the original article.

This is the authors' version of the following article:

Songjun Li, Yi Ge, Sergey A Piletsky and Anthony Turner, A Zipper-Like On/Off-Switchable Molecularly Imprinted Polymer, 2011, Advanced Functional Materials, (21), 17, 3344-3349.

which has been published in final form at: http://dx.doi.org/10.1002/adfm.201100593

Copyright: Wiley-VCH Verlag Berlin http://www.wiley-vch.de/publish/en/

Postprint available at: Linköping University Electronic Press http://urn.kb.se/resolve?urn=urn:nbn:se:liu:diva-71385 


\title{
A Zipper-Like 'On/Off'-Switchable Molecularly Imprinted Polymer
}

\author{
Songjun Li, Yi Ge, Sergey A. Piletsky, and Anthony P. F. Turner
}

Molecular imprinting has been the subject of extensive recent investigation. Dubbed a 'from-key-to-lock' technology, molecular imprinting provides a straightforward route to prepare molecular recognition materials (i.e., molecularly imprinted polymers: MIPs) comparable to natural antibodies, but with improved properties. ${ }^{[1,2]}$ Impressive progress has been made in this field due to a better understanding of the mechanisms involved in forming selective imprints, and also the employment of new methods of synthesis, which have led to significant advances in the production of materials for various analytical and separation applications. ${ }^{[3,4]}$

One recent challenge in molecular imprinting has been the aspiration of achieving controllable molecular recognition. ${ }^{[5,6]}$. Thanks to rapid progress in the field of controlled release, this objective is being facilitated by borrowing the 'smart' properties from controlled release technology. ${ }^{[7]}$ One typical case is poly( $N$-isopropylacylamide) (PNIPAm)-based MIPs, which demonstrate controllable molecular recognition resulting from the thermosensitive hydrophilicity/hydrophobicity of PNIPAm (transition temperature, $\left.\sim 32{ }^{\circ} \mathrm{C}\right) .{ }^{[8]}$ The hydrophilicity of PNIPAm decreases with rising temperature. Thus, PNIPAm-based MIPs in water exhibit lower molecular recognition abilities at elevated temperatures due to the increased hydrophobicity, which inhibits access to the imprinted networks. However, in most cases this inverse molecular recognition ability does not find significant practical application given the low molecular recognition capability and slow kinetics at low temperature. Furthermore, it is challenging to identify the polymer's hydrophilic/hydrophobic properties during the adsorption process. On the contrary, the fabrication of MIPs having positive 'on/off'-switchable functions would generate substantial potential for applications. To date, this need has not been met.

\footnotetext{
Dr. S. Li, Dr. Y. Ge, Prof. S.A. Piletsky and [*] Prof. A.P.F. Turner

Cranfield Health, Vincent Building, Cranfield University

Cranfield, Bedfordshire, MK43 0AL, UK

Email: a.p.turner@cranfield.ac.uk (A.P.F. Turner)

[*] Prof. A.P.F. Turner

Biosensors \& Bioelectronics Centre, IFM, Linköping University, SE-58183, Linköping, Sweden

Email: anthony.turner@liu.se (A.P.F. Turner)

[**] The authors thank the Research Directorate-General of European Commission for supporting this work under the framework of Marie Curie Actions (PIIF-GA-2009-236799). Thanks also should be expressed to the National Science Foundation of China (No. 21073068).
} 
We recently described a zipper-like nanoreactor with controllable reactivity. ${ }^{[9]}$ This nanoreactor was composed of $\mathrm{Ag}$ nanoparticles and a polymer matrix made of poly(acrylamideco-2-acrylamide-2-methylpropanesulfonic acid) (PAAm-co-PAMPS), where the interactions within the interpolymer complexes acted as a switch for the nanoreactor. In detail, the zipper-like PAAm-PAMPS interaction led to opening or closing of the polymeric networks and thus controllable reactivity. The formation of the PAAm-PAMPS complexes at relatively low temperatures caused shrinking of the polymeric networks and inhibited access of the reactant to the catalytic $\mathrm{Ag}$ nanoparticles, resulting in weak reactivity. In contrast, the interpolymer interactions between PAAm and PAMPS were disrupted at higher temperatures, causing significant reactivity.

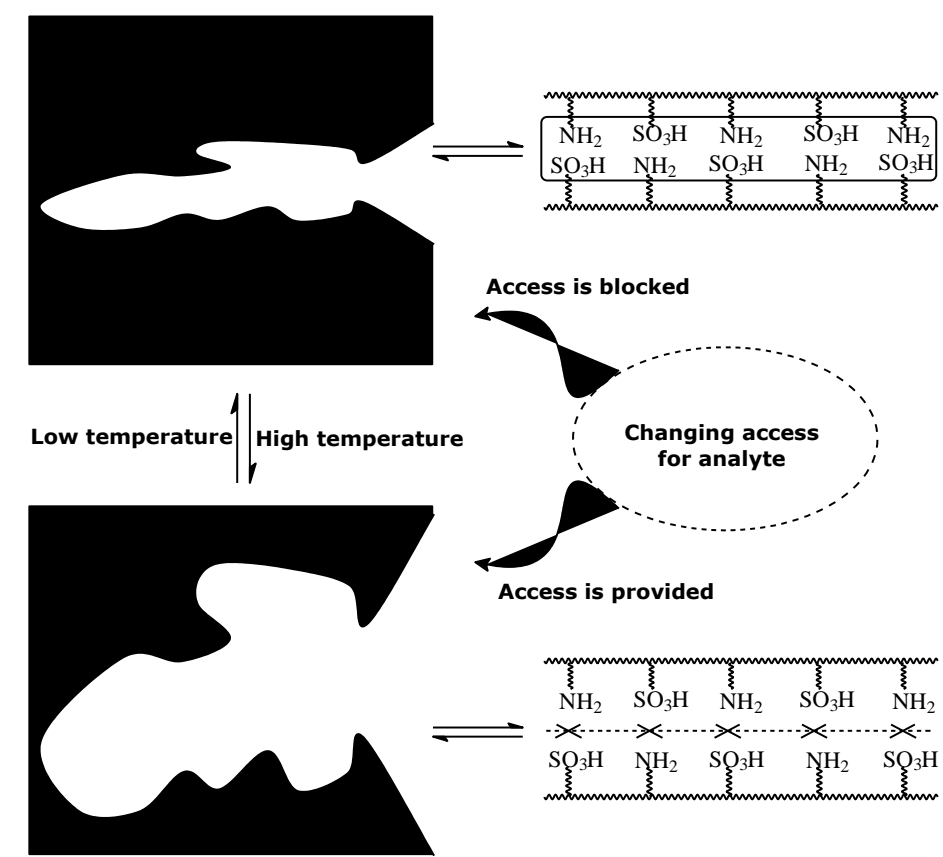

Scheme 1. Proposed mechanism for the zipper-like imprinted polymer.

Building on this work, we report herein the first zipper-like imprinted polymer with 'on/off'-switchable functions (i.e., 'MIP-S'). As proposed in Scheme 1, this MIP-S displayed poor molecular recognition at relatively low temperatures due to the interpolymer complexation between PAAm and PAMPS, which restricted access of the analyte to the imprinted networks. On the contrary, the MIP-S demonstrated significant molecular recognition at relatively high temperatures resulting from the dissociation of the interpolymer complexes, which allowed the analyte to gain access to the polymer interior. In this way, this unique MIP-S demonstrated an 'on/off'-switchable molecular recognition ability. 
$S$-naproxen was selected as the template to prepare the MIP-S, because of its compatibility with water and the availability of sophisticated imprinting methods. ${ }^{[10]}$ To meet the demand of an optimised 'imprint', ${ }^{[1]}$ stoichiometric template-monomers and AAm-MAPS interactions have to be employed. Figures 1 and 2 display the UV spectra altered as a function of the AAm/MAPS ratio and the monomers/template ratio, respectively. As shown in Figure 1, the titration of AAm into AMPS caused a shift in the UV absorption band of AAm. The shift achieved a maximal value when the titrated AAm reached the critical amount (corresponding to a ratio of $1.2 \mathrm{~mol} / \mathrm{mol}$ of AAM/AMPS). It is assumed that the AAm-AMPS interactions were saturated by the stoichiometric titration and the ratio of AAm to AMPS was optimised. Using this optimised AAm-to-AMPS ratio, the optimal amount of template was further tested. As shown in Figure 2, the shift of the UV absorption band reached the maximal value when the template-monomers ratio reached 1: $1.48: 1.23$. Thus, $0.32 \mathrm{~g}(4.45 \mathrm{mmol})$ of AAm and $0.77 \mathrm{~g}$ (3.71 mmol) AMPS were used to prepare the MIP-S, when $0.69 \mathrm{~g}(3.0 \mathrm{mmol})$ of $S$-naproxen was adopted (Scheme 2). For comparison, two control polymers, i.e., the corresponding non-imprinted and a conventional imprinted polymers (named 'NIP-S' and 'MIP-C', respectively), were also prepared under comparable conditions. NIP-S was prepared by using the same composition as MIP-S, except that no template was used during the preparation. MIP-C had the same composition as MIP-S, but in which AMPS was replaced with the same amount of AAM.

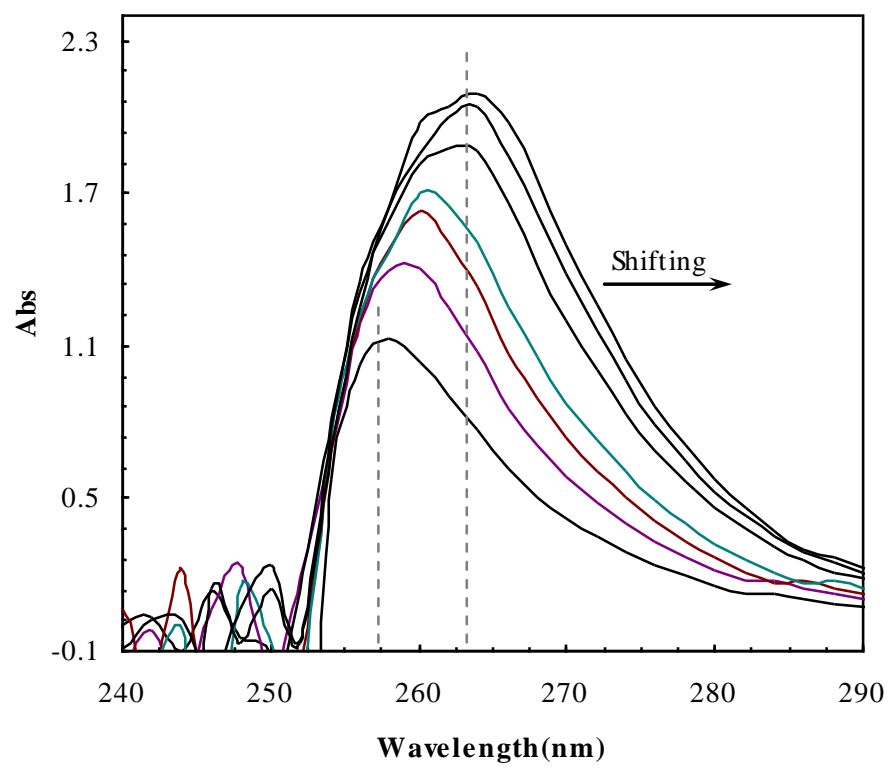

Figure 1. UV spectra of the titration of AAM with AMPS (where AAm (0.5 mM) was titrated into AMPS (0.5 mM). 


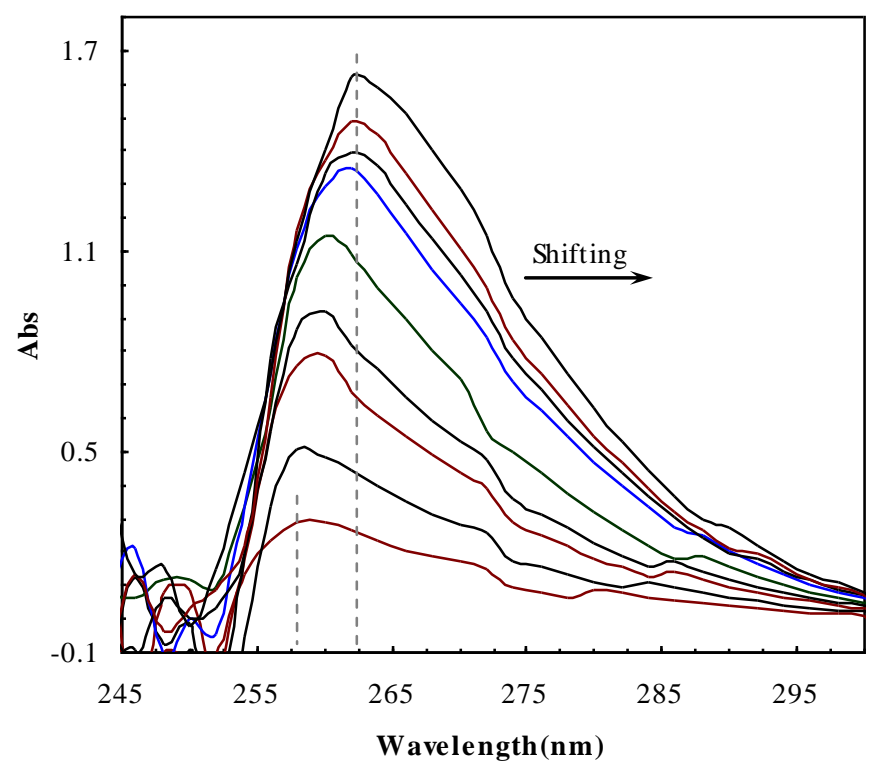

Figure 2. UV spectra of the titration of monomers with $S$-naproxen (The stoichiometric ratio of AAm/AMPS (AAm, 0.5 $\mathrm{mM})$ was titrated into $S$-naproxen $(0.5 \mathrm{mM})$.

FTIR spectra were used to track the imprinting process during the preparation of MIP-S, as shown in Figure 3 (where the MIP-S precursor is the MIP-S system where the imprinted $S$-naproxen was not removed from the polymer matrix). Three characteristic peaks $\left(3400-3600,2900-3100, \sim 1750 \mathrm{~cm}^{-1}\right)$ and one broad fingerprint band (1000-1600 $\left.\mathrm{cm}^{-1}\right)$ appeared in the spectra of both MIP-S systems. These characteristic peaks can be attributed to the stretching vibration of $\mathrm{O}-\mathrm{H}(\mathrm{N}-\mathrm{H}), \mathrm{C}-\mathrm{H}$ and $\mathrm{C}=\mathrm{O} .{ }^{[12]}$ The fingerprint band may arise from $\mathrm{C}-\mathrm{N}$ and $\mathrm{C}-\mathrm{C}$ bonds and their rotation. ${ }^{[13]}$ For comparison, we also included the FTIR spectra of two control polymers to Figure 3. The MIP-S precursor, MIP-S and NIP-S all displayed the broad fingerprint band at $1000-1600 \mathrm{~cm}^{-1}$, but MIP-C failed to show this. It is thus clear that both the MIP-S and NIP-S systems had PAMPS in their polymeric networks. ${ }^{[14]}$ As further noted, the MIP-S precursor incorporated both characteristic peaks of NIP-S and S-naproxen in the FTIR spectrum, thereby implying its complicated composition. After washing, the spectrum of the MIP-S precursor (viz. MIP-S) became comparable to that of NIP-S. It is therefore clear that the imprinting of $S$-naproxen occurred during the preparation, as expected.

Temperature-programmed desorption (TPD) experiments were conducted, in order to further characterise the 'imprint'. As shown in Figure 4, both MIP-S and MIP-C demonstrated significant separation abilities for $S$-naproxen and $R$-naproxen (i.e., the enantiomer of $S$-naproxen), but NIP-S did not show such capability. This demonstrates that the interaction between MIP-S and MIP-C with 
$S$-naproxen is specific and capable of separation of the template and its enantiomer. In conjunction with the FTIR characterisation, this result confirms the specificity of the imprinted polymer for $S$-naproxen and facilitates further studies on its switchable properties.

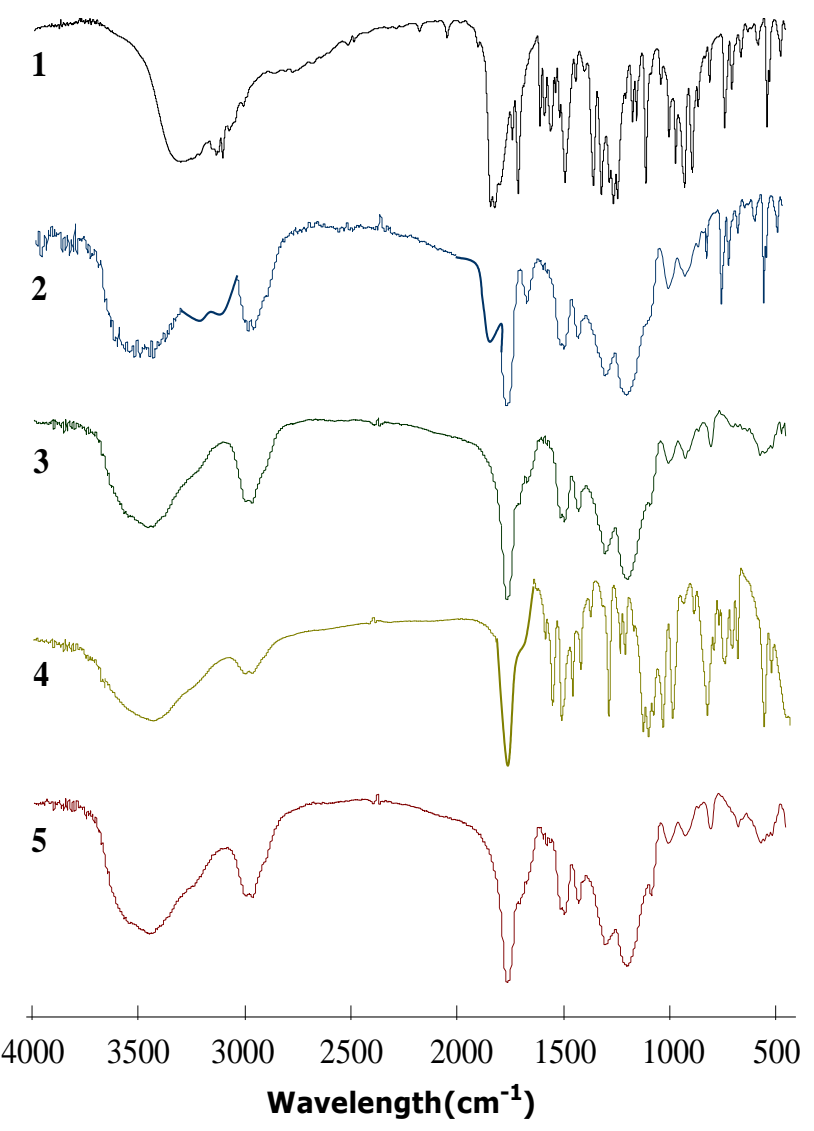

Figure 3. FTIR spectra of the prepared polymers (1: S-naproxen; 2: MIP-S precursor; 3: MIP-S; 4: MIP-C; 5: NIP-S)

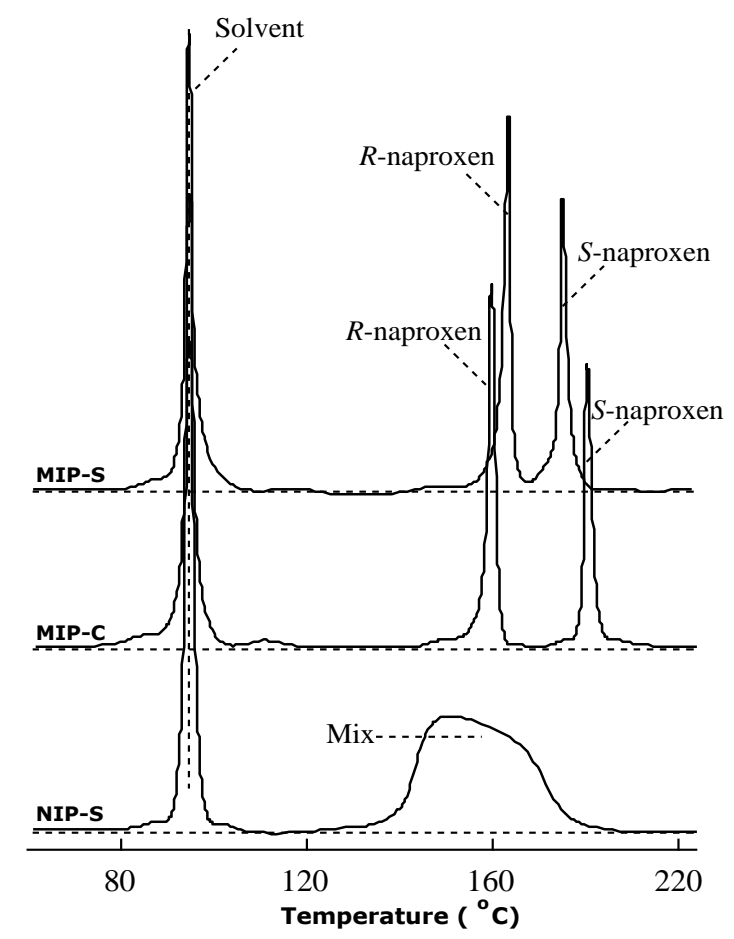

Figure 4. TPD profiles of the prepared polymers. 
The switchable interaction between PAAm and PAMPS was studied as a function of temperature, as shown in Figure 5. By a comparison between both 'smart' polymers (i.e., MIP-S and NIP-S) and the conventional MIP-C, the contribution of the PAAm-PAMPS interaction can be reflected by water intake $\left(W_{\mathrm{c}}\right) .{ }^{[15]} \mathrm{MIP}-\mathrm{S}$ and NIP-S displayed significant dependence on temperature, relative to MIP-C. The $W_{c}$ of MIP-S and NIP-S increased with elevated temperature and the major increase appeared at $\sim 27-35{ }^{\circ} \mathrm{C}$. Below this region, the $W_{\mathrm{c}}$ of MIP-S and NIP-S demonstrated a low $W_{c}$ associated with the interpolymer complexation between PAAm and PAMPS, which inhibited swelling of the polymeric networks (Scheme 1). In contrast, above this temperature range, the $W_{c}$ of MIP-S and NIP-S dramatically increased, in response to the dissociation of the interpolymer interaction, which caused swelling of the polymeric networks. This demonstrates the switchable properties of MIP-S and NIP-S.

The adsorption properties of these polymers are illustrated in Figure 6. In order to test the switchable molecular recognition, two representative temperatures, i.e., 40 and $20{ }^{\circ} \mathrm{C}$ (either higher or lower than the thermosensitive region of MIP-S and NIP-S, i.e., $\sim 27-35{ }^{\circ} \mathrm{C}$ ), were selected for a contrastive study. At $40{ }^{\circ} \mathrm{C}$, MIP-S was comparable to MIP-C and displayed significant molecular recognition for the imprint species (Figure $6 \boldsymbol{a}$ ). In contrast, at $20{ }^{\circ} \mathrm{C}$, MIP-S was more like NIP-S, which exhibited poor molecular recognition towards its template (Figure $6 \boldsymbol{b}$ ). MIP-S demonstrated switchable molecular recognition abilities, as expected.

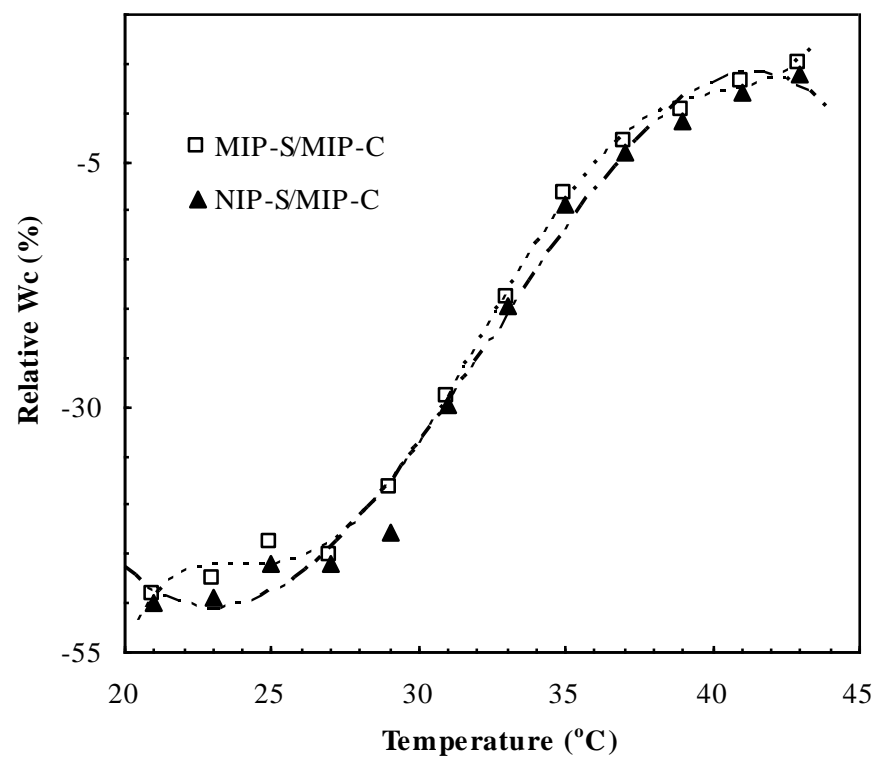

Figure 5. Water intake of the prepared polymers. 

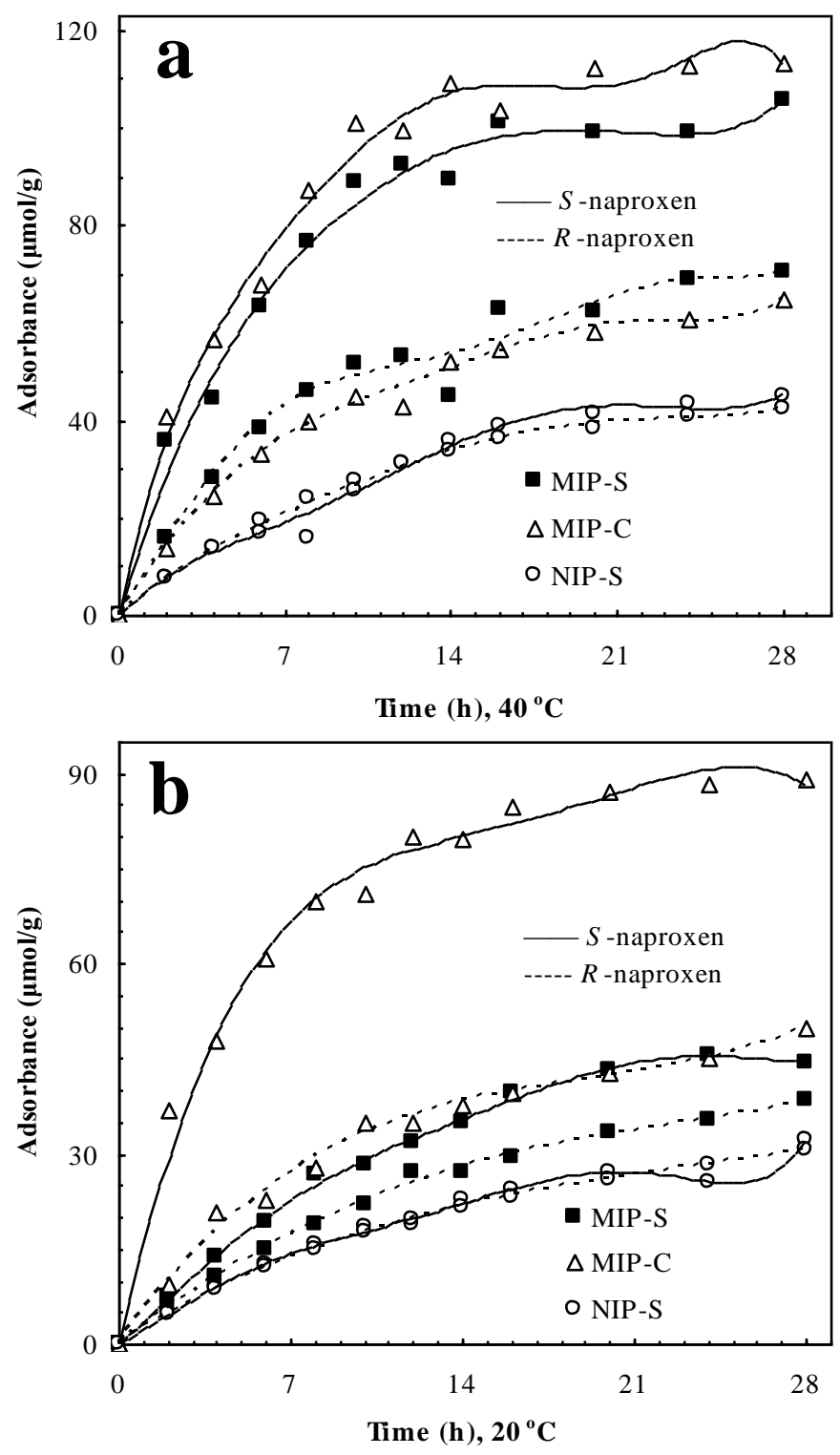

Figure 6. Adsorption curves of the prepared polymers

Electrochemical studies were also conducted in order to ascertain the thermo-switching mechanism, as shown in Figure 7. It is known that the potential to oxidise or reduce a binding molecule depends on the binding constant. A high binding constant requires more energy to overcome the binding, thereby causing a larger redox potential. Thus, dynamic desorbing cyclic voltammetry (DCV) may provide valuable information on the binding interaction between MIPs and their template. ${ }^{[16]}$ By considering the thermosensitive region, 20 and $40{ }^{\circ} \mathrm{C}$ were further selected for the contrastive study. The imprint molecule (viz. S-naproxen) that was bound to MIP-S at $20{ }^{\circ} \mathrm{C}$ exhibited a reduction peak at $-1068 \mathrm{mV}$ (Figure $7 \boldsymbol{c})$. In contrast, this reduction peak at $40{ }^{\circ} \mathrm{C}$ shifted to a lower potential (i.e., $-1202 \mathrm{mV}$ ) (Figure 7d). MIP-S displayed a stronger interaction with the imprint molecule at $40{ }^{\circ} \mathrm{C}$ than at $20{ }^{\circ} \mathrm{C}$. 
For comparison, Figure 7 (i.e., $a, b, e$, and $f$ ) also included the reduction potentials of the imprint molecule from two control polymers. The reduction potential of the imprint molecule that was bound to MIP-S at $40{ }^{\circ} \mathrm{C}$ was nearly comparable to that from MIP-C (-1202 vs. $\left.-1221 \mathrm{mV}\right)$. The reduction potential of the imprint molecule that was bound to MIP-S at $20{ }^{\circ} \mathrm{C}$ became as low as that from NIP-S (-1068 vs. $-1029 \mathrm{mV})$. This strongly suggests that MIP-S underwent thermo-switching interactions with the imprint molecule. Again, this result can be attributed to the unique zipper-like networks. The switchable mechanism by MIP-S regulated access of the analyte to the imprinted networks, thereby enabling the switched binding.
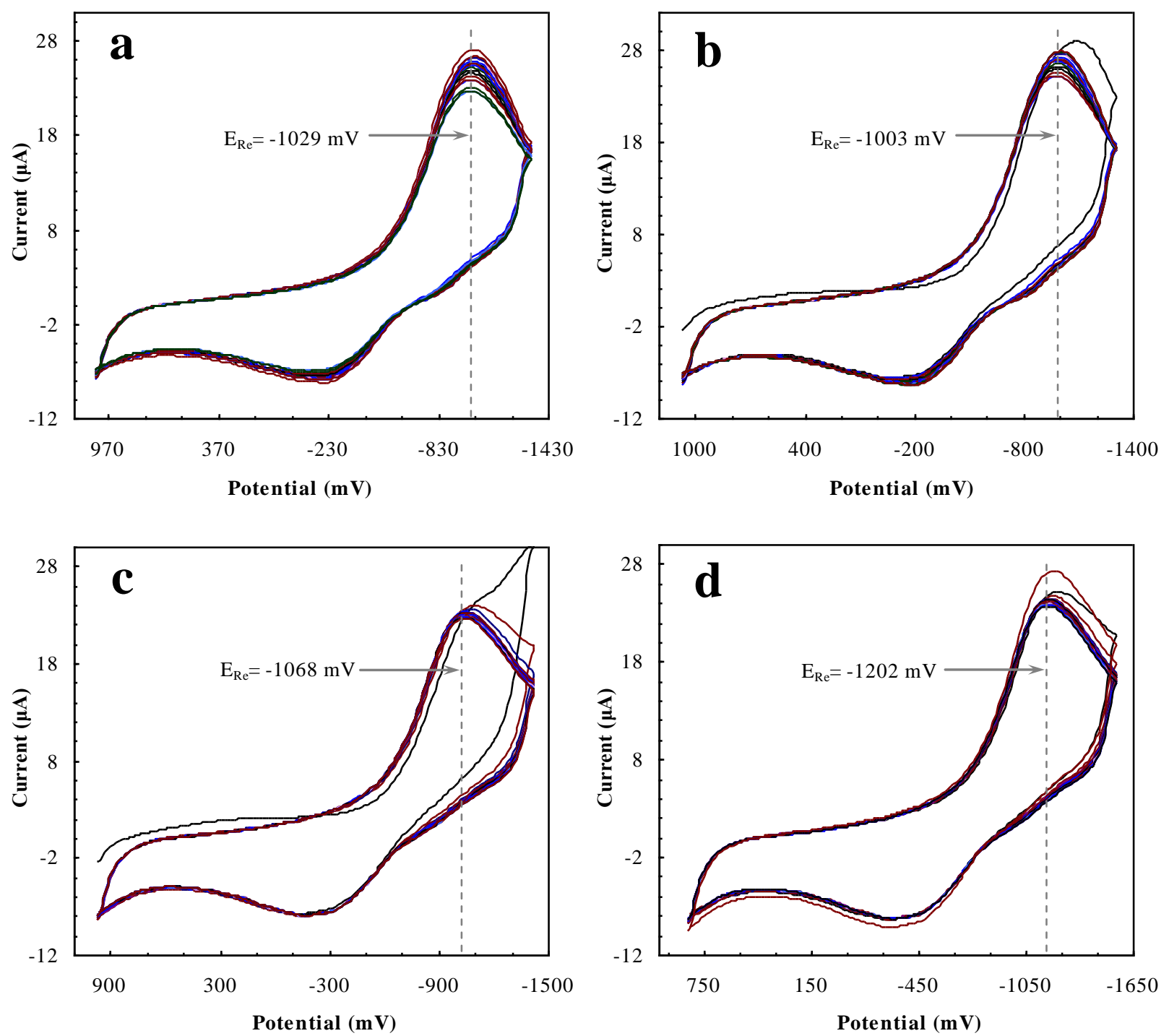

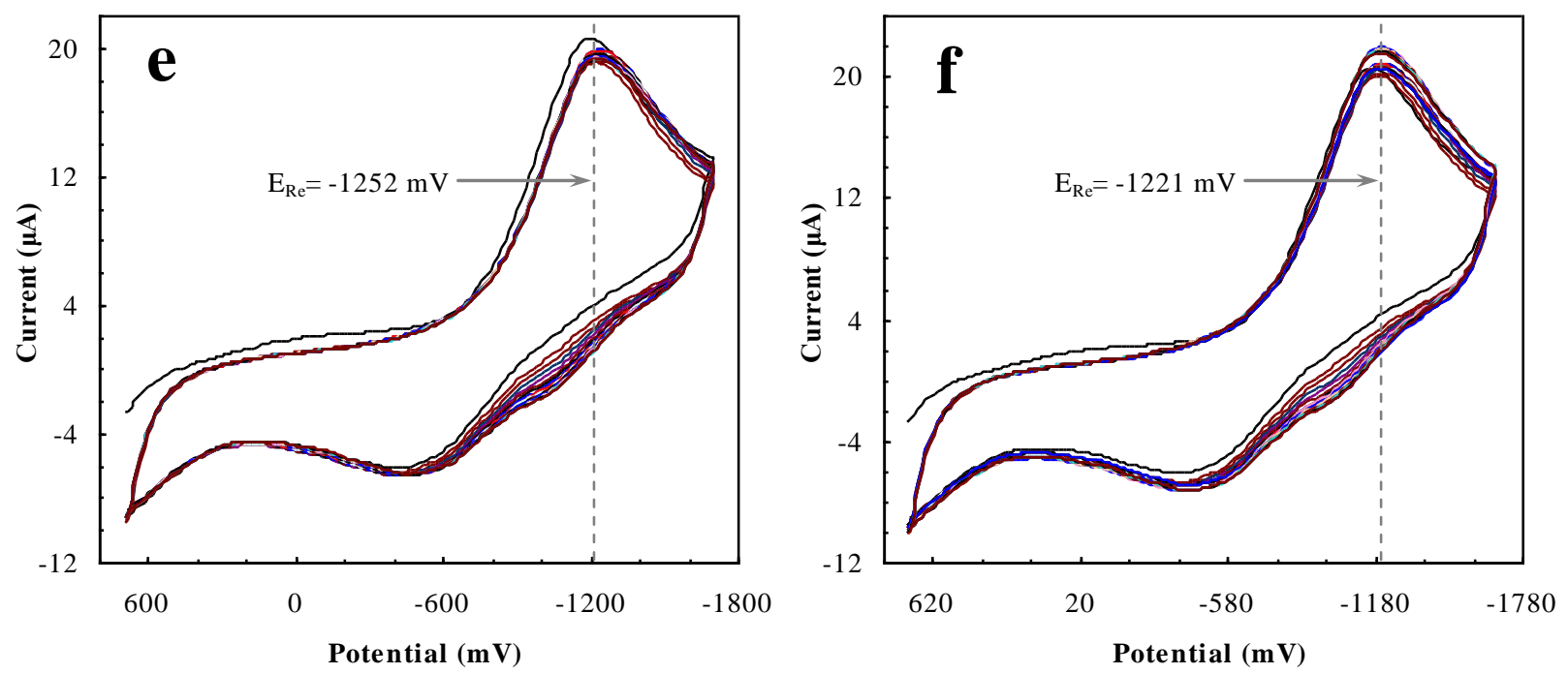

Figure 7. DCV profiles of the template molecule from the prepared polymers

(a: NIP-S at $20{ }^{\circ} \mathrm{C} ; b$ : NIP-S at $40{ }^{\circ} \mathrm{C} ; c$ : MIP-S at $20{ }^{\circ} \mathrm{C} ; d$ : MIP-S at $40{ }^{\circ} \mathrm{C} ; e$ : MIP-C at $20{ }^{\circ} \mathrm{C} ; f$ : MIP-C at $40{ }^{\circ} \mathrm{C}$ )

In conclusion, the first zipper-like imprinted polymer capable of 'on/off'-switchable functions is reported herein. This polymer showed marginal recognition ability towards the imprint species under low temperature conditions (such as $20^{\circ} \mathrm{C}$ ). However, at higher temperatures, it presented significant molecular recognition abilities. It is suggested that a new generation of MIPs with 'on/off'-switchable functions can be developed by using such novel designs and methods.

\section{Experimental Section}

Preparation of imprinted polymers: Unless otherwise noted, chemicals were obtained from Aldrich (Sigma-Aldrich Company Ltd, Dorset, UK) and Fisher (Fisher Scientific UK Ltd, Loughborough, UK) and used as received without further purification. The preparation of molecularly imprinted polymers was based on the optimised designs, as previously discussed. $S$-naproxen (0.69 g), ethylene glycol dimethacrylate (EGDMA; $7.0 \mathrm{~mL}), 2$ 2'-azobis(isobutyronitrile) (AIBN; $0.3 \mathrm{~g}$ ) and a stoichiometric amount of AAm and AMPS (relative to the template) were dissolved in dimethylsulfoxide (15mL) to form a homogeneous system. After being deoxygenated with sonication and nitrogen, the mixed system was irradiated by ultraviolet light $(365 \mathrm{~nm})$ overnight. The resulting polymers were crushed and extracted with dimethylsulfoxide containing $20 \%$ acetic acid using a Soxhlet apparatus for $24 \mathrm{~h}$. The products were profusely washed with ethanol and acetic acid and then dried at room temperature. TPD: Using gas chromatography (TCD) and a data processing system, polymers (10 mg) were placed 
into an online U-shaped quartz tube (4 mm I.D.). After $10 \mu \mathrm{L}$ of analytes $\left(1 \mu \mathrm{mol} \mathrm{mL}^{-1}\right.$ dimethylsulfoxide) was pre-adsorbed by these polymers, the U-shaped tube was heated under a nitrogen flow $\left(30 \mathrm{~mL} \mathrm{~min}{ }^{-1} ; 0.3 \mathrm{MPa}\right)$ at $10{ }^{\circ} \mathrm{C} \mathrm{min}^{-1}$ from room temperature to the temperature where the analyte desorbed. The desorbing signal was recorded by the data processing system.

Switchable interactions: Dried samples (in triplicate) were immersed in PBS (pH 7.0) until equilibrium was reached. After the water adhered on external surfaces was blotted with a cloth, the samples were weighed $\left(W_{\mathrm{t}}\right)$ and then dried in a vacuum vessel $\left(W_{\mathrm{d}}\right)$. By a comparison between both 'smart' polymers (i.e., MIP-S and NIP-S) and the conventional MIP-C, the contribution of the PAAm-PAMPS interaction can be reflected by water intake $\left(W_{\mathrm{c}}\right):^{[15]}$

$$
W_{c}=\left[\left(\frac{W_{t}-W_{d}}{W_{d}}\right)_{T}-\left(\frac{W_{t}-W_{d}}{W_{d}}\right)_{N T}\right] \times 100 \%
$$

Where, $T$ represents the 'smart' polymers and $N T$ indicates the non-thermosensitive polymer.

Adsorption test: The adsorption properties of the prepared polymers were evaluated using a batch format. ${ }^{[9]}$ The initial concentration of $S$-naproxen was $1.0 \mu \mathrm{mol} \mathrm{mL} \mathrm{mL}^{-1}$ (totally $15 \mathrm{~mL} \mathrm{PBS}$; pH 7.0). The solid content of polymers was $5.0 \mathrm{mg} \mathrm{mL}^{-1}$ in each test. The change of the analyte concentration was spectrophotometrically monitored at $331 \mathrm{~nm}$. The adsorption amount of these polymers was obtained from the mass balance of analytes and based on the average value of identical triplicate runs. DCV: Using an electrochemical workstation equipped with a three-electrode configuration (Pt-working and counter electrodes; $\mathrm{Ag}^{+} / \mathrm{AgCl}$ - ref.) (LK9806) (Lanlike, China), polymers (10 mg) pre-absorbed with $c a .1 \mu \mathrm{mol}$ template were placed into a cuvette encircled by a diffusion-eliminated sonication apparatus (supporting electrolyte: $0.01 \mathrm{mmol} \mathrm{mL}^{-1} \mathrm{KNO}_{3} ; 10 \mathrm{~mL} \mathrm{PBS}(\mathrm{pH} 7.0)$ ). The transiently desorbed template was rapidly scanned by the workstation up to 20 cycles until a stable dynamic cyclic voltammogram was achieved (scanning range, $c a$. 1.2 -1.2 $\mathrm{V}$; scanning rate, 0.25 $\left.V \cdot \mathrm{s}^{-1}\right)$.

Keywords: Molecularly imprinted polymers $\cdot$ molecular recognition $\cdot$ host-guest interaction 


\section{References}

[1] A. Nematollahzadeh, W. Sun, C. S. A. Aureliano, D. Lütkemeyer, J. Stute, M. J. Abdekhodaie, A. Shojaei, B. Sellergren, Angew. Chem. Int. Ed. 2011, 50, 495-498.

[2] R. N. Liang, D. A. Song, R. M. Zhang, W. Qin, Angew. Chem. Int. Ed. 2010, 49, 2556-2559.

[3] Y. J. Zhao, X. W. Zhao, J. Hu, J. Li, W. Y. Xu, Z. Z. Gu, Angew. Chem. Int. Ed. 2009, 48, $7350-7352$.

[4] A. Poma, A. P. F. Turner, S. A. Piletsky, Trends Biotechnol. 2010, 28, 629-637.

[5] C. B. Gong, M. H. W. Lam, H. X. Yu, Adv. Funct. Mater. 2006, 16, 1759-1767.

[6] Z. Chen, Z. Hua, L. Xu, Y. Huang, M. Zhao, Y. Li, J. Mol. Recognit. 2008, 21, 71-77.

[7] Z. Chen, L. Xu, Y. Liang, M. Zhao, Adv. Mater. 2010, 22, 1488-1492.

[8] V. Abbate, N. Frascione, S. S. Bansal, J. Polym. Sci. A 2010, 48, 1721-1731.

[9] S. Li, Y. Ge, A. Tiwari, S. Cao, Small, 2010, 21, 2453-2459.

[10]W. Li, S. Li, Adv. Polym. Sci. 2007, 206, 191-210

[11]B. C. G. Karlsson, J. O’Mahony, J. G. Karlsson, H. Bengtsson, L. A. Eriksson, I. A. Nicholls, J. Am. Chem. Soc. 2009, 131, 13297-13304.

[12]B. Singh, N. Sharma, N. Chauhan, Carbohyd. Polym. 2007, 69, 631-643.

[13] S. Li, S. Gong, Adv. Funct. Mater. 2009, 19, 2601-2606.

[14]C. Wang, T. Wang, Q. Wang, Polymer 2010, 51, 4836-4842.

[15] S. Li, A. Tiwari, Y. Ge, D. Fei, Adv. Mater. Lett. 2010, 1, 4-10.

[16] S. Li, S. Gong, J. Phys. Chem. B 2009, 113, 16501-16507. 